\title{
Tecnura
}

\section{Evaluación del riesgo por deslizamiento de una ladera en la ciudad de Tijuana, México}

\section{Landslide risk assessment of a slope in Tijuana city, Mexico}

\author{
Aldo Onel Oliva González ${ }^{1}$ Romel Jesús Gallardo Amaya²
}

Fecha de recepción: 25 de mayo de 2017

Fecha de aceptación: 28 de noviembre de 2017

Cómo citar: Oliva G., A.O. y Gallardo A., R.J. (2018). Evaluación del riesgo por deslizamiento de una ladera en la ciudad de Tijuana, México. Revista Tecnura, 22(55), 34-50. https://doi.org/10.14483/22487638.12063

\section{Resumen}

Contexto: La reducción del riesgo y prevención de eventos desastrosos producidos por deslizamientos de tierra en laderas urbanas requiere de una evaluación integral que considere los factores condicionantes y desencadenantes de carácter natural y antrópico. Dicha evaluación constituye una valiosa herramienta de prevención/mitigación para las comunidades en riesgos y para las autoridades competentes.

Método: En este trabajo de investigación se estudió y aplicó una metodología general para evaluar el riesgo por deslizamiento de una ladera urbana, considerando la interrelación entre la amenaza y vulnerabilidad física existentes en la zona de estudio. La amenaza se determinó a través de métodos probabilísticos y determinísticos; mientras que la vulnerabilidad de los elementos expuestos se obtuvo considerando los tipos de edificaciones y su distribución espacial, el estado de integridad estructural, la profundidad de las cimentaciones y el volumen probable de la masa de terreno inestable.

Resultados: Se obtuvieron factores de seguridad por debajo de los aceptables para garantizar la estabilidad de la ladera objeto de estudio, y los factores de valuación del análisis cualitativo indican que la ladera es inestable y requiere de atención urgente. Lo anterior confirma y valida la alta probabilidad de ocurrencia de deslizamientos en la zona, obtenida de los registros históricos.

Conclusiones: Se encontró que el riesgo por deslizamiento de la ladera es alto debido a la elevada probabilidad de ocurrencia, con tres posibles direcciones de movimiento que pueden impactar varias edificaciones que se encuentran en la zona. La evaluación constituye una herramienta de trabajo para las instituciones y autoridades que tienen como misión reducir el riesgo por deslizamientos, como forma de prevenir y mitigar eventos generadores de desastres.

Palabras clave: amenaza, análisis de estabilidad, deslizamiento de ladera, riesgo, vulnerabilidad.

\begin{abstract}
Context: Risk reduction and prevention of disasters events produced by landslides on urban slopes, requires an integral assessment considering conditioning and triggering natural and human factors. Such an assessment is a valuable prevention and mitigation tool for communities under risk and also for authorities involved in the process.

Method: In this research, a general methodology for the assessment of landslides on an urban slope was
\end{abstract}

1 Ingeniero civil, especialista en Geotecnia, doctor en Ingeniería. Docente e investigador de la Universidad de las Californias Internacional. Tijuana, México. Contacto: aldo.oliva@udc.edu.mx

2 Ingeniero civil, especialista en Ingeniería Ambiental, magíster en Geotecnia. Docente e investigador del Departamento de Ingeniería Civil de la Universidad Francisco de Paula Santander Ocaña. Ocaña, Colombia. Contacto: rjgallardoa@ufpso.edu.co 
studied and applied, considering the relationship between hazard and physical vulnerability in the zone of study. Hazard was determined by probabilistic methods, whereas vulnerability of the exposed elements was obtained taking into account two kinds of buildings and their spatial distribution, their structural integrity state, their foundation depth and the unstable terrain probable mass volume.

Results: Safety factors were obtained under allowable levels to warrant stability of the slope under study, and valuation factors of the qualitative analysis indicate that the slope is unstable and that requires urgent maintenance. This confirms and validates the high probability of occurrence in the zone, obtained from historic records.

Conclusions: It was found that landslide risk in the slope is high due to the high probability of its occurrence, with three possible movement directions that may impact on several buildings located in the zone. Assessment constitutes a work tool for institutions and authorities related with risk reduction due to landslides, as a way of prevent and mitigate disaster prone events.

Keywords: hazard, stability analysis, landslide, risk, vulnerability.

\section{INTRODUCCIÓN}

Para que en una ladera se generen deslizamientos se debe materializar la acción conjunta de factores geológicos, hidrológicos y geomorfológicos, y la alteración de estos por procesos geodinámicos, presencia de vegetación y de actividad antrópica, así como por la frecuencia e intensidad de fenómenos naturales como terremotos y precipitaciones. Además, en la ocurrencia de los deslizamientos se presenta mucha incertidumbre debido a que estos pueden tener diversas formas de falla, velocidad de movimiento y diversas condiciones de los materiales de la ladera. A esto se suma el hecho de que los parámetros y modelos matemáticos usados para el análisis de estabilidad, también tienen incertidumbre (Morgenstern, 1997).

Lo anterior hace que el valor del factor de seguridad de una ladera, obtenido como forma de evaluar su estabilidad, pueda ser más o menos confiable en función de considerar (o no) los mencionados factores de incertidumbre.

El riesgo producido por deslizamientos depende de la amenaza y la vulnerabilidad y puede expresarse según la ecuación (1).

$$
R_{i e_{t}}=f\left(\mathrm{~A}_{i} \cdot \mathrm{V}_{e}\right)_{t}
$$

La anterior ecuación significa que, si conocemos la amenaza dada por la probabilidad de que se presente un deslizamiento con cierta intensidad durante un periodo de tiempo específico, y la vulnerabilidad, entendida como la predisposición de los elementos expuestos a sufrir daños ante la ocurrencia del suceso, se puede determinar el riesgo, como la probabilidad de que se presenten pérdidas en los elementos expuestos.

La probabilidad de ocurrencia de un deslizamiento puede variar entre 0 y 1 . Donde el valor cero expresa la imposibilidad de que ocurra el evento y uno es la certeza. Dicha probabilidad se puede expresar de acuerdo con lo recomendado por IUGS Working Group on Landslides Committee on Risk Assessment (1997), en función de:

- La cantidad de deslizamientos en un año que pueden ocurrir en el área objeto de estudio.

- La probabilidad de que en una ladera se presenten deslizamientos en un periodo dado.

- Las fuerzas desequilibrantes que exceden las fuerzas estabilizadoras en términos de probabilidad, sin considerar una frecuencia anual (factor de seguridad).

En este trabajo se aplican métodos y procedimientos de análisis para evaluar el riesgo generado 
por el posible deslizamiento de una ladera urbana en la ciudad de Tijuana, México.

\section{Antecedentes}

Los deslizamientos de laderas en la ciudad de Tijuana se presentan repetidamente en distintas zonas del área urbana, causando la destrucción total y parcial de gran cantidad de viviendas e infraestructuras. Debido al crecimiento urbano no planificado e incontrolado de las últimas décadas, más del $30 \%$ de la población vive en terrenos inestables y poco resistentes ubicados en lomeríos con laderas de grandes pendientes. Esto, unido a la complejidad geológica y geotécnica y a la fuerte actividad tectónica de la región, hace que la inestabilidad de laderas sea una amenaza permanente y que el riesgo de ocurrencia de movimientos de masas de suelo y rocas alcance niveles difíciles de predecir (Oliva et al., 2014).

\section{METODOLOGÍA}

La zona objeto de estudio es la urbanización Lomas Conjunto Residencial, seleccionada debido a la aparición, en agosto de 2011, de una fractura en forma de grieta semicircular que parece indicar el inicio de un deslizamiento de ladera (figura 1).

El presunto movimiento del terreno, afectó directamente varias estructuras ubicadas en las inmediaciones de la fractura (figura 2). Según reportes de la Dirección Municipal de Protección Civil de Tijuana (DMPCT), existen antecedentes de movimientos de laderas en sitios cercanos, que han dañado severamente viviendas de urbanizaciones ubicadas en los sectores sur y suroeste de la zona de estudio.

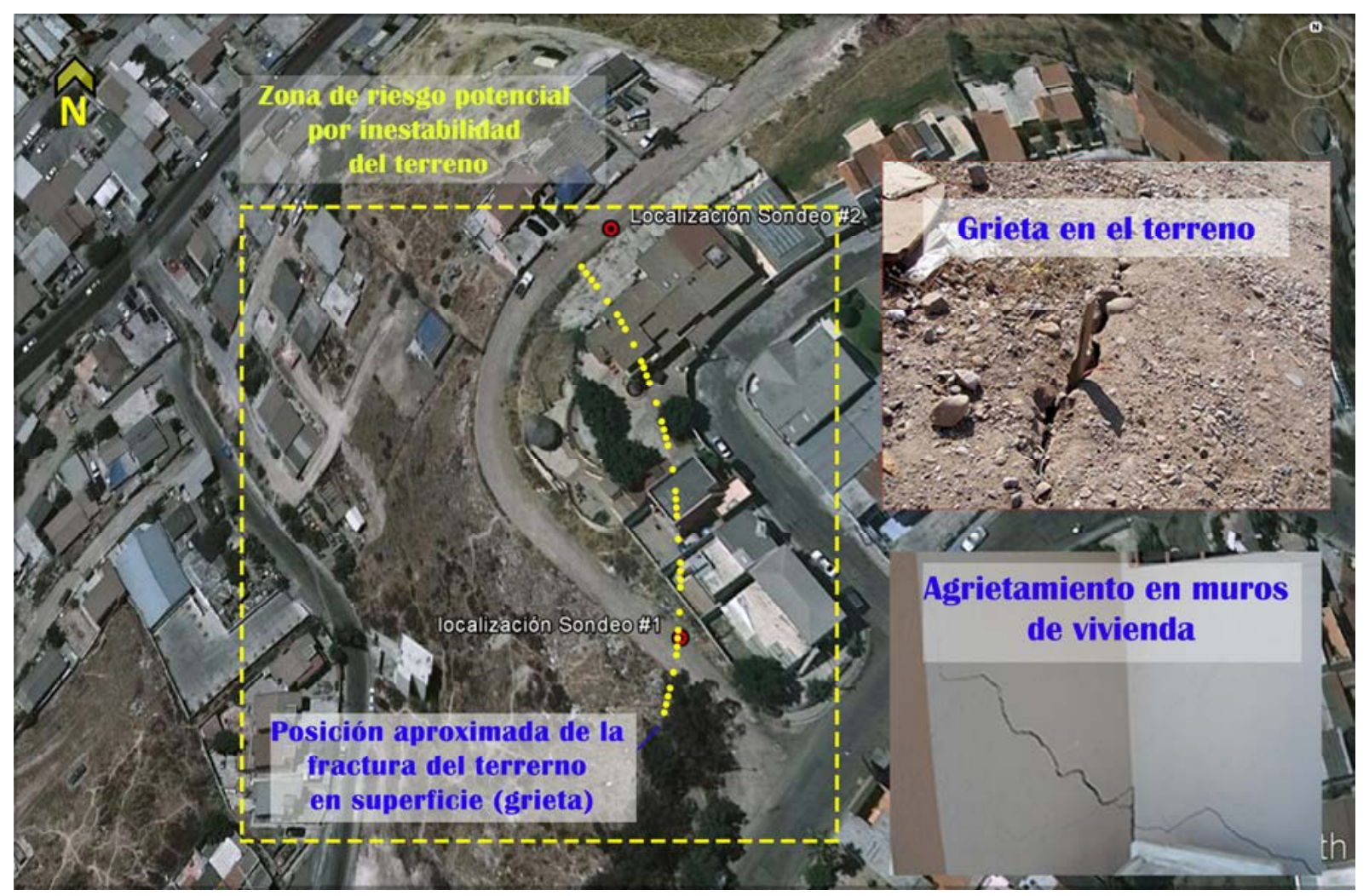

Figura 1. Zona de riesgo potencial por deslizamiento

Fuente: adaptado de Google Earth. 


\section{Ubicación y características del sitio}

El área de estudio se ubica en el centro-sur de la ciudad de Tijuana y en el sector norte de la urbanización Lomas Conjunto Residencial, en la delegación La Mesa (figura 3). Se localiza en las coordenadas $32^{\circ} 29^{\prime} 15,65^{\prime \prime}$ y $116^{\circ} 57^{\prime} 29,34^{\prime \prime}$ (WGS84).

\section{Caracterización del área de estudio}

En esta etapa se realizaron los siguientes trabajos:

- Levantamiento topográfico del sitio para caracterizar geométricamente el área afectada (o de potencial afección) y determinar con precisión: alturas, pendientes y formas de los taludes y laderas que fueron considerados en el posterior análisis de la estabilidad. Además, se realizó una cartografía de los agrietamientos geotécnicos y estructurales existentes.
- Estudios geológicos y geotécnicos que incluyeron la exploración y muestreo para caracterizar geológica y geotécnicamente el terreno en el área de estudio. Se obtuvieron perfiles estratigráficos del subsuelo y parámetros físico-mecánicos de los materiales. Además, se realizaron estudios geofísicos para corroborar algunos de los resultados obtenidos en las pruebas de campo y laboratorio.

- Instrumentación de dos sondeos para el monitoreo y control de movimientos horizontales mediante sonda inclinométrica.

\section{Análisis de la estabilidad}

En esta etapa se realizaron análisis cuantitativo y cualitativo de la estabilidad de la ladera objeto de estudio. El primero, mediante métodos de equilibrio límite y tensodeformacionales; y el segundo, utilizando factores de valuación.

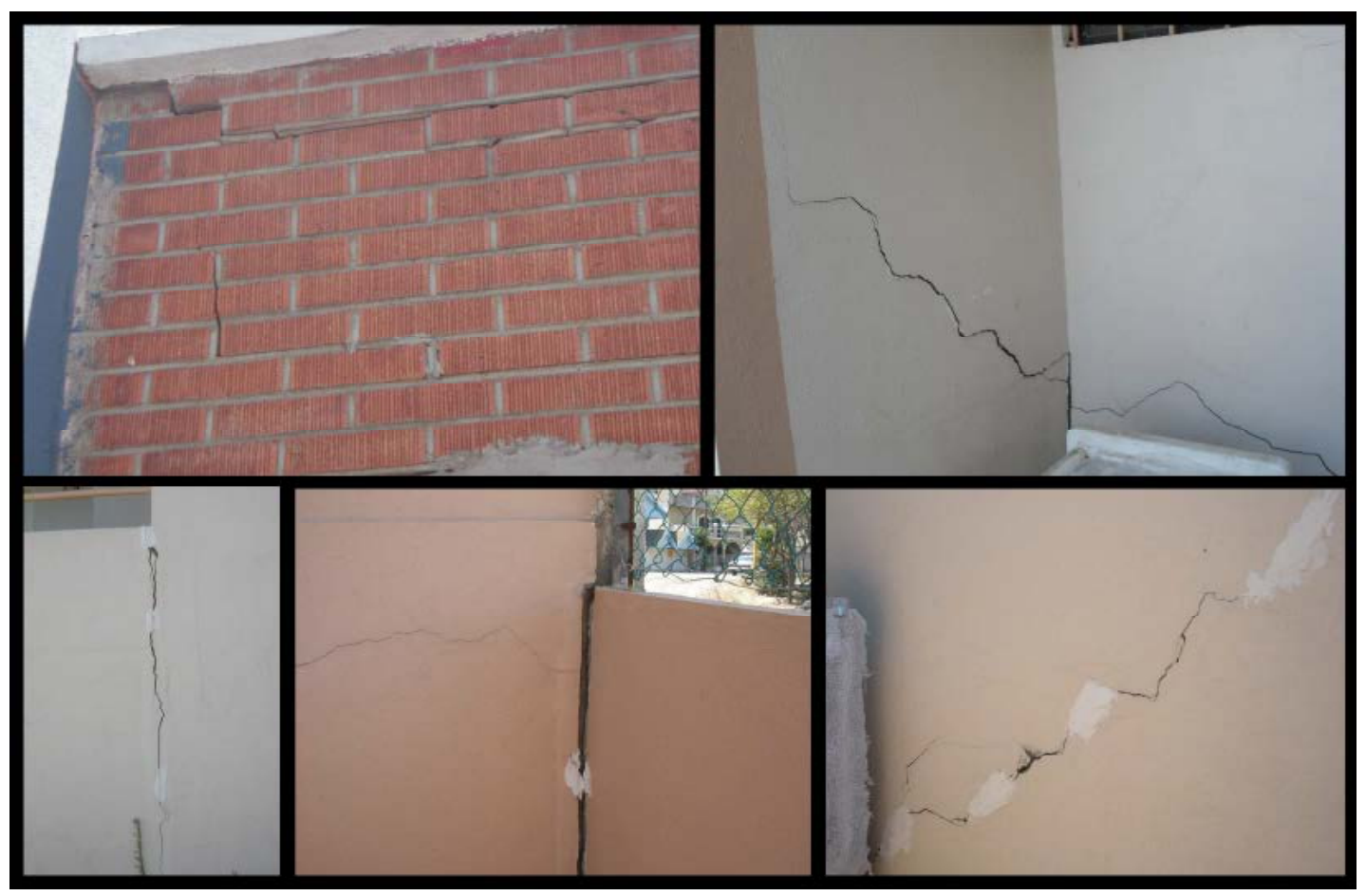

Figura 2. Daños en edificaciones ubicadas en el área de estudio

Fuente: elaboración propia. 


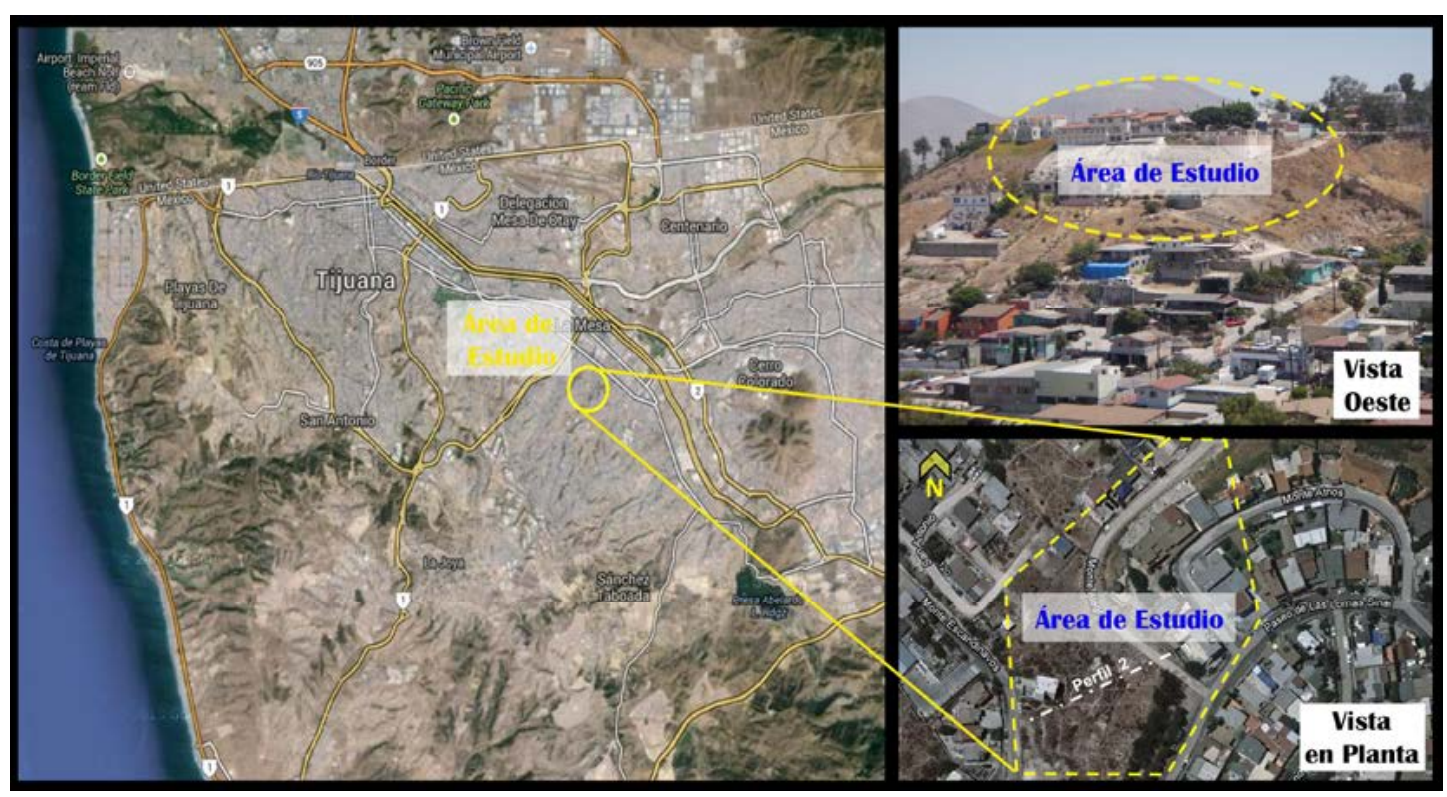

Figura 3. Ubicación del área de estudio

Fuente: elaboración propia.

\section{Evaluación del riesgo}

Para la evaluación, el riesgo fue considerado como una función de la amenaza, dada por la interrelación susceptibilidad/probabilidad de ocurrencia; y de la vulnerabilidad obtenida considerando la probabilidad de que el deslizamiento impacte a los elementos expuestos analizados y produzca daños en sus estructuras.

\section{RESULTADOS}

\section{Topografía}

Se realizó un levantamiento topográfico de la zona, se establecieron puntos de control para verificar movimientos del terreno y se obtuvieron perfiles topográficos representativos para generar los modelos geométricos utilizados en el análisis de estabilidad. En la figura 4 se muestra la planta topográfica y los perfiles utilizados en el análisis de la estabilidad.

\section{Geología}

En el área de estudio, las Formaciones San Diego y Otay se encuentran sobre la Formación Rosarito Beach y, en ocasiones, se observa la Formación Lindavista en las capas superficiales.

La Formación San Diego (Plioceno-Pleistoceno) se caracteriza por areniscas micáceas estratificadas con tonalidades gris claro y amarillo naranja. Intercaladas con estas areniscas se encuentran arenas con tamaños de partícula que van de finos a medios y conglomerados constituidos por gravas y boleos de tamaño variable.

La Formación Otay se considera del Plioceno, aunque algunos autores le han asignado edades miocénicas, como el equivalente lateral del sur de la Formación Rosarito Beach. Descansa sobre una superficie erosionada de rocas del Eoceno, está constituida por sedimentos de origen no marinos y reflejan la deposición en una cuenca alargada norte-sur. En el área de Tijuana, la Formación se caracteriza por la presencia de tobas arenáceas de 


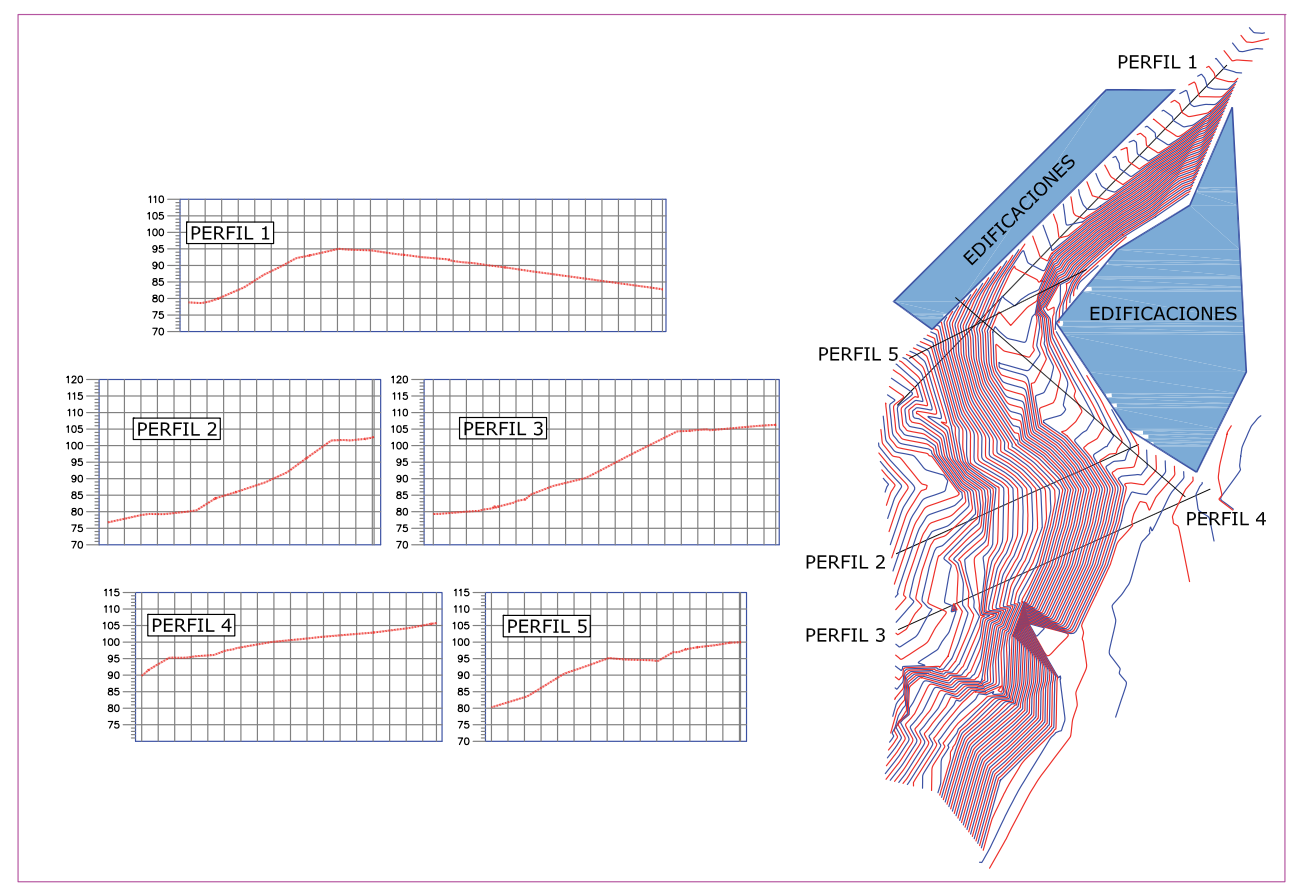

Figura 4. Planta y perfiles topográficos del área de estudio

Fuente: elaboración propia.

grano fino con arcilla muy impermeable de color café claro a gris que, a cierta profundidad, producen bloques litificados muy erráticos del mismo material.

La Formación Lindavista (Pleistoceno) está compuesta por areniscas y conglomerados de color café-rojizo; un alto porcentaje de clastos de material metavolcánico con cantidades menores de material riolítico y cuarcitas. Las arenas depositadas cerca de la línea de costa presentan una interestratificación de conglomerados con cementante ferruginoso, principalmente limonita.

\section{Tectónica}

La actividad sismotectónica en el norte de Baja California muestra cierta complejidad debido al contacto de tipo transcurrente entre las placas tectónicas de Norteamérica y Pacífico, así como a la existencia de varios grupos de fallas activas en la región (Suárez-Vidal et al., 1991; Correa y Alfaro, 2011).
En el área objeto de estudio, el marco tectónico está condicionado por fallas que atraviesan las sierras peninsulares, como Agua Blanca, Ojos Negros y el sistema San Miguel-Vallecitos (figura 5), cuya actividad afecta directamente a la zona urbana de Tijuana (Cruz-Castillo, 2002; Cyment, 1979; Hungr, 1997). La respuesta más común al marco tectónico dominante en la zona es la intensa actividad sísmica de forma perceptible e imperceptible (microsismicidad). Esta última puede ser indicativo de un proceso de movimientos en diferentes niveles de la corteza terrestre, que cuando rebasan ciertas magnitudes son agentes disparadores de los deslizamientos.

\section{Geotecnia}

Se realizaron dos sondeos mecánicos a una profundidad de 25 metros cada uno, en los cuales se hicieron pruebas de penetración estándar (SPT, por su sigla en inglés) y se tomaron muestras en cada metro de avance (figura 6). Dichas muestras fueron 


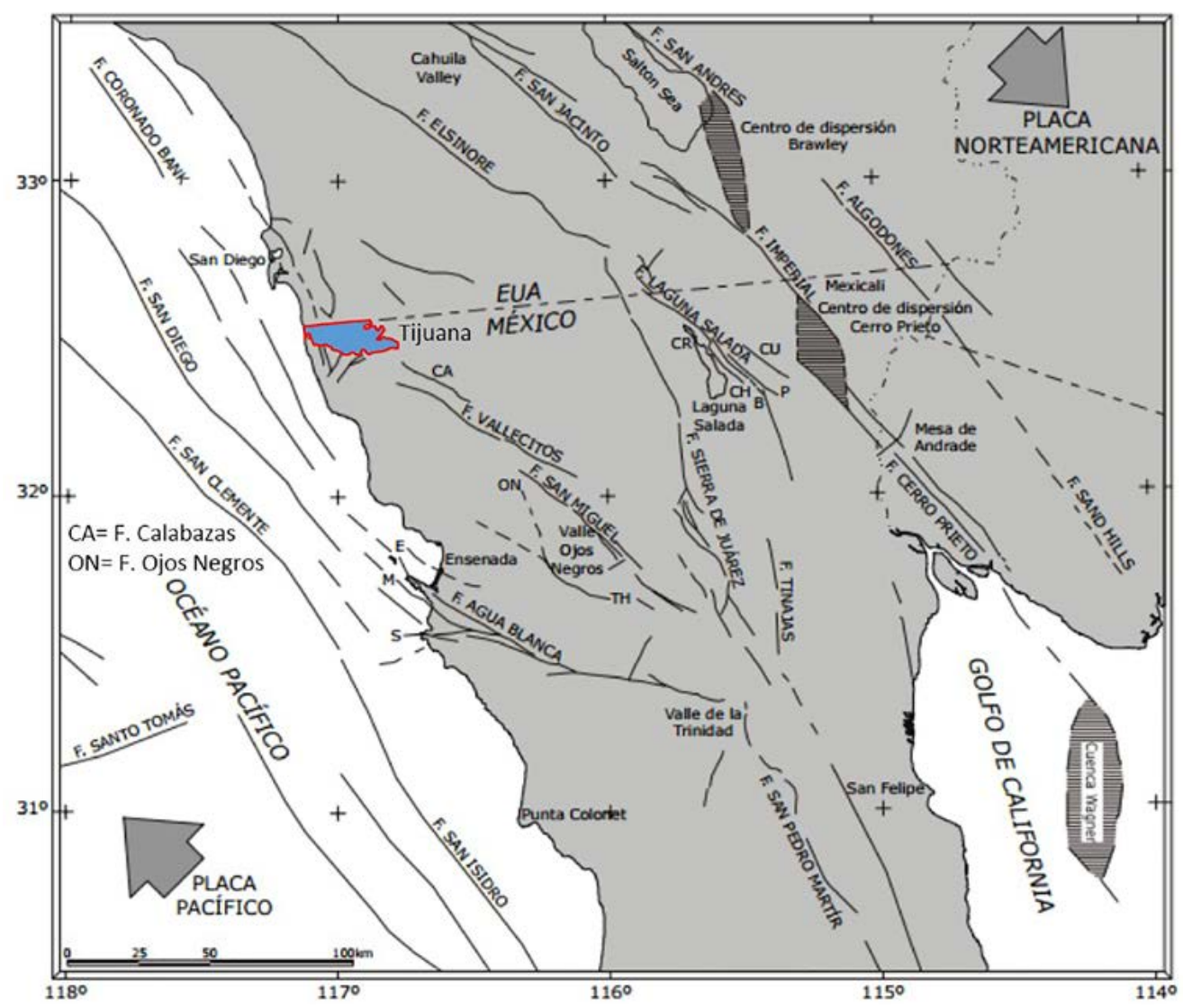

Figura 5. Situación sismotectónica de la ciudad de Tijuana

Fuente: modificado de Acosta-Chang et al. (2009) (Fondo Nacional para la Prevención de Desastres Naturales [FOPREDEN], 2012; Cruz-Castillo, 2002).

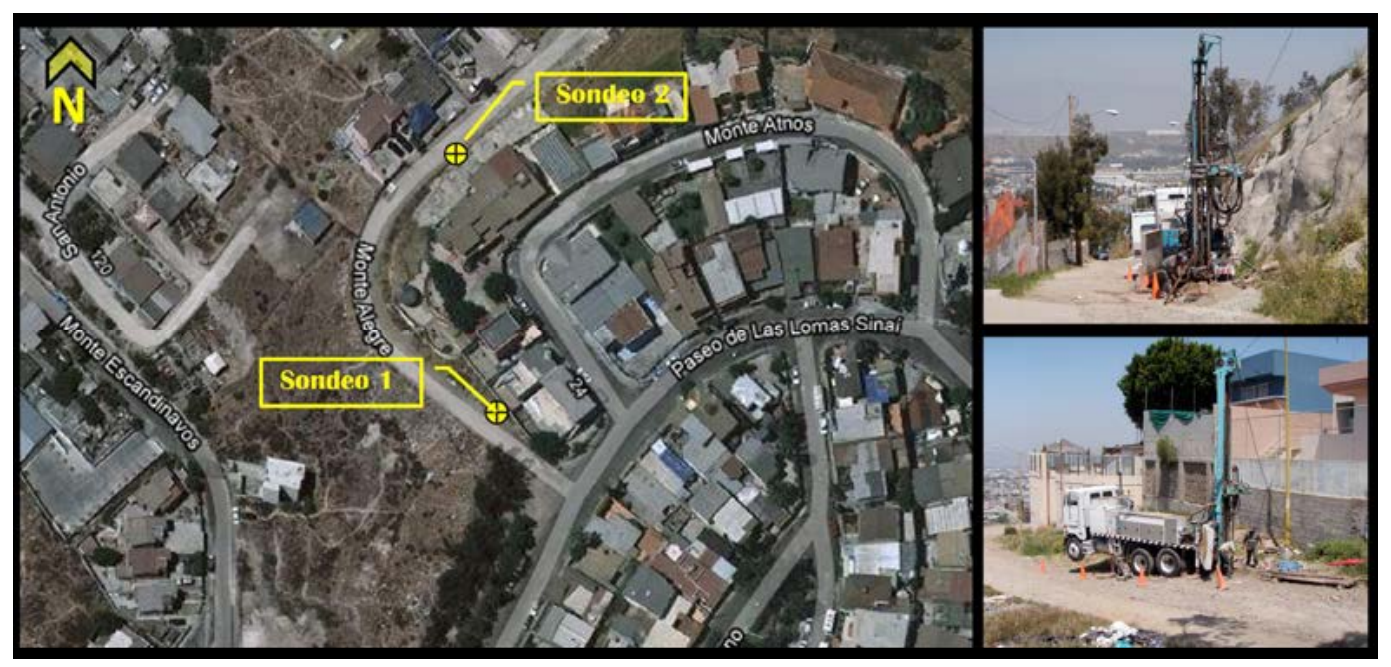

Figura 6. Ubicación de los sondeos

Fuente: elaboración propia. 
sometidas a diferentes pruebas de laboratorio para obtener los parámetros físico-mecánicos de los materiales predominantes en el sitio.

La tabla 1 muestra las características principales de los materiales que conforman el perfil estratigráfico del área de estudio.

\section{Análisis cuantitativo de la estabilidad}

Tuvo por finalidad determinar los factores de seguridad críticos para las condiciones más desfavorables de equilibrio y sus respectivas superficies de rotura; así como los desplazamientos y vectores de movimientos del terreno en la ladera objeto de estudio. Se consideraron los esfuerzos estáticos producidos por el peso propio del terreno y la condición dinámica debido a los sismos o microsismicidad característicos de la zona.

Para considerar el efecto de la sismicidad y evaluar su influencia en la formación de los agrietamientos existentes en la zona de estudio, se consideraron las aceleraciones generadas por sismos de magnitudes superiores a 3 grados, registrados en la región dos meses antes de la aparición de la fractura, así como las amplificaciones de dichas aceleraciones por efecto de sitio (Acosta-Chang et al., 2009).

La tabla 2 muestra los factores de seguridad mínimos obtenidos considerando los esfuerzos estáticos y dinámicos en la ladera.

Tabla 1. Perfil estratigráfico del área de estudio

\begin{tabular}{|c|c|c|c|}
\hline Estrato & Espesor $(\mathrm{m})$ & Descripción & Parámetros físico-mecánicos \\
\hline Material 1 & $7,00-13,00$ & $\begin{array}{l}\text { Conglomerados con boleos de } \\
\text { tamaño variable en una matriz } \\
\text { arenosa de grano medio a } \\
\text { grueso (Formaciones Lindavista } \\
\text { y San Diego). }\end{array}$ & $\begin{array}{l}\text { Peso volumétrico }=19-20 \mathrm{kN} / \mathrm{m}^{3} \\
\text { Ángulo de fricción }=35-38^{\circ} \\
\text { Cohesión }=40-50 \mathrm{kN} / \mathrm{m}^{2} \\
\mathrm{E}=291257-296160 \mathrm{kN} / \mathrm{m}^{2} \\
\mathrm{G}=100027-101989 \mathrm{kN} / \mathrm{m}^{2} \\
\mathrm{v}=0,45\end{array}$ \\
\hline Material 2 & $4,00-12,00$ & $\begin{array}{l}\text { Tobas arenáceas de color café } \\
\text { claro y gris; con arena fina, } \\
\text { limos y arcilla (Formación Otay). }\end{array}$ & $\begin{array}{l}\text { Peso volumétrico }=18-20 \mathrm{kN} / \mathrm{m}^{3} \\
\text { Ángulo de fricción }=25-35^{\circ} \\
\text { Cohesión }=20-25 \mathrm{kN} / \mathrm{m}^{2} \\
\mathrm{E}=2450682-3176374 \mathrm{kN} / \mathrm{m}^{2} \\
\mathrm{G}=845039-1095403 \mathrm{kN} / \mathrm{m}^{2} \\
\mathrm{v}=0,45\end{array}$ \\
\hline Material 3 & Más de 14,00 & $\begin{array}{l}\text { Tobas arenáceas compactas (For- } \\
\text { mación Otay) }\end{array}$ & $\begin{array}{l}\text { Peso volumétrico }=18-20 \mathrm{kN} / \mathrm{m}^{3} \\
\text { Ángulo de fricción }=25-35^{\circ} \\
\text { Cohesión }=35-50 \mathrm{kN} / \mathrm{m}^{2} \\
\mathrm{E}=24849075-51216220 \mathrm{KN} / \mathrm{m}^{2} \\
\mathrm{G}=8568758-17660799 \mathrm{KN} / \mathrm{m}^{2} \\
\mathrm{v}=0.45\end{array}$ \\
\hline
\end{tabular}

Fuente: elaboración propia.

Tabla 2. Factores de seguridad mínimos

\begin{tabular}{ccc}
\hline No. de perfil & Factor de seguridad estático & Factor de seguridad dinámico \\
\hline 1 & 2.160 & 1.094 \\
\hline 2 & 1.930 & 0.994 \\
\hline 3 & 2.288 & 1.136 \\
\hline 5 & 2.295 & 1.285 \\
\hline
\end{tabular}

Fuente: elaboración propia. 
Los resultados indican que los perfiles 1,2 y 5 alcanzan condiciones críticas de equilibrio ante el efecto de la sismicidad, siendo la condición más desfavorable de inestabilidad la del perfil 2. La figura 7 muestra el modelo de análisis del perfil 2, donde se observan las superficies de deslizamientos calculadas. La zona de color verde representa la masa de terreno con mayor probabilidad de falla (factor de seguridad mínimo) y la de color rojo, otras posibles superficies de deslizamiento.

Un análisis tensodeformacional en el perfil 2 permitió obtener la variación del factor de seguridad en función de la aceleración sísmica y el comportamiento de los desplazamientos en la zona donde se encuentra la grieta (figura 8).

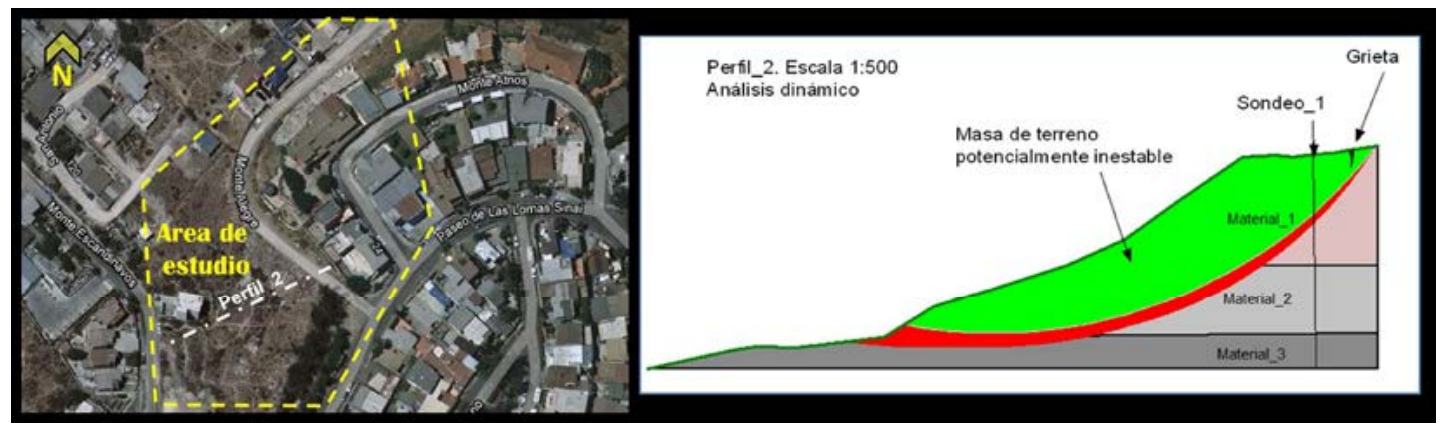

Figura 7. Resultados del análisis de estabilidad en el perfil 2

Fuente: elaboración propia.

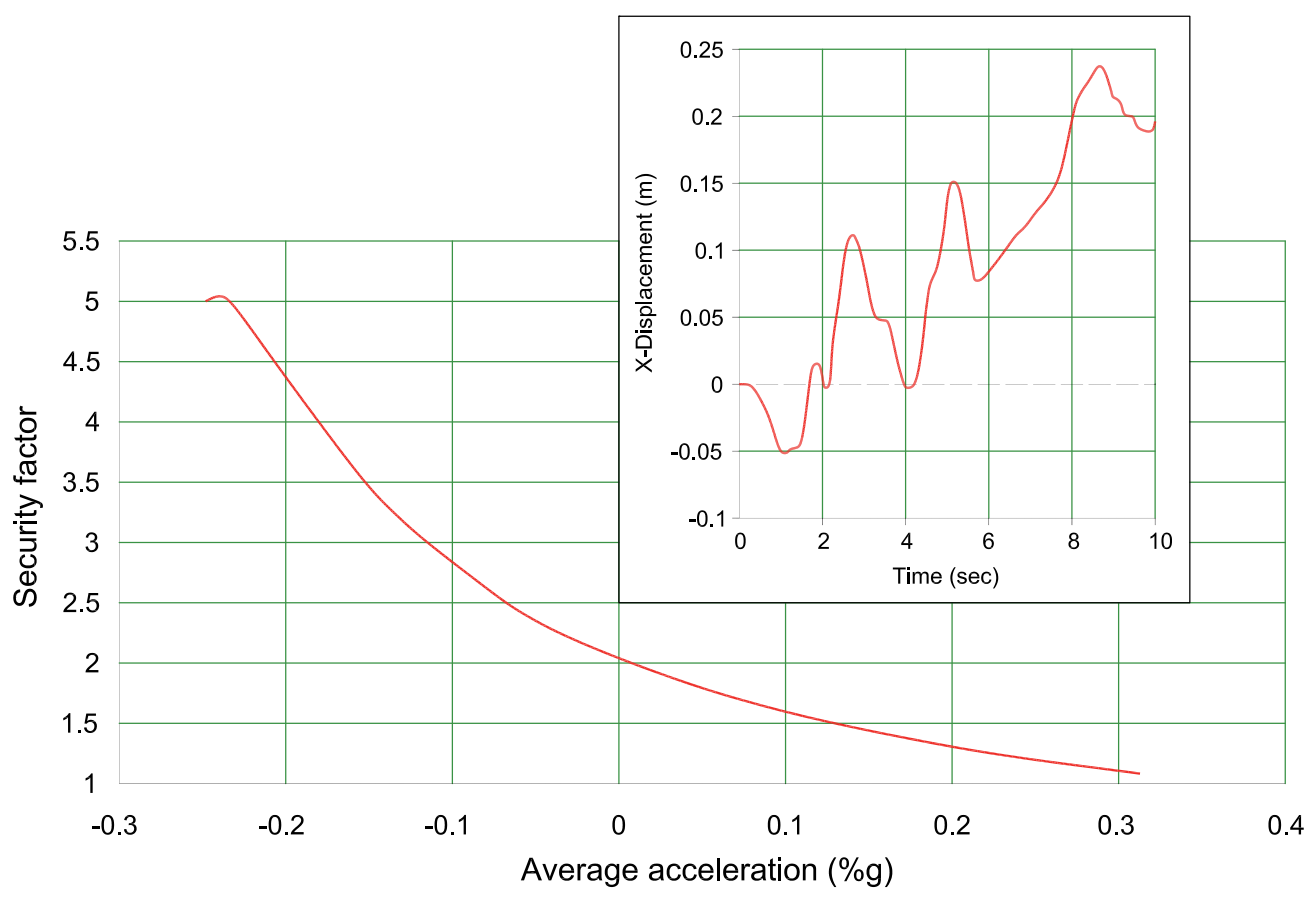

Figura 8. Factor de seguridad y desplazamientos en el entorno de la grieta (perfil 2)

Fuente: elaboración propia. 


\section{Análisis cualitativo de la estabilidad}

Se realizó con el objetivo de evaluar la susceptibilidad a la ocurrencia de deslizamientos en la zona de estudio, considerando la influencia de diversos factores condicionantes y desencadenantes de la inestabilidad. Para ello, se utilizaron los factores de valuación que se describen en la tabla 3 (Cuanalo, Oliva y González, 2007; Cuanalo, Oliva y Gallardo, 2012).

A los factores de valuación se le asignaron intervalos de valores ponderados comprendidos entre 0 y 1 , que indican su efecto sobre la estabilidad (Cuanalo, Oliva y González, 2007). El valor 0 corresponde a un efecto nulo o mínimo sobre la estabilidad (no influye o influye muy poco); y el 1 corresponde al de mayor impacto sobre la estabilidad (influye significativamente). La tabla 4 muestra los intervalos y niveles de influencia utilizados para evaluar la estabilidad.

Los resultados del análisis cualitativo de la estabilidad en la ladera de la zona de estudio se muestran en la tabla 5 .

Tabla 3. Factores de valuación considerados en el análisis

\begin{tabular}{|c|c|c|c|}
\hline Factor de valuación & \multicolumn{2}{|c|}{ Concepto } & Obtenidos en función de: \\
\hline \multirow{9}{*}{$\begin{array}{l}\text { Dependiente de las carac- } \\
\text { terísticas intrínsecas de la } \\
\text { ladera }\end{array}$} & \multicolumn{2}{|c|}{ Morfología y topografía } & Forma e inclinación de la ladera. \\
\hline & \multirow{2}{*}{$\begin{array}{l}\text { Mecánica de } \\
\text { suelos }\end{array}$} & Suelos friccionantes & Inclinación de la ladera y ángulo de fricción interna. \\
\hline & & $\begin{array}{l}\text { Suelos finos (limos y } \\
\text { arcillas) }\end{array}$ & $\begin{array}{l}\text { Inclinación de la ladera, altura, peso volumétrico y } \\
\text { resistencia no drenada. }\end{array}$ \\
\hline & \multirow{2}{*}{\multicolumn{2}{|c|}{ Hidrogeología }} & Inclinación de la ladera y grado de saturación del suelo. \\
\hline & & & Espesor del suelo. \\
\hline & \multirow{4}{*}{\multicolumn{2}{|c|}{ Vegetación }} & Tipo de vegetación. \\
\hline & & & Densidad de follaje. \\
\hline & & & Área cubierta. \\
\hline & & & Tipo de raíz. \\
\hline \multirow{7}{*}{$\begin{array}{l}\text { Dependientes de las condi- } \\
\text { ciones regionales del sitio }\end{array}$} & \multicolumn{2}{|l|}{ Terremotos } & Coeficiente sísmico. \\
\hline & \multirow{3}{*}{\multicolumn{2}{|c|}{ Erosión y socavación }} & Características del suelo superficial. \\
\hline & & & Área de la cuenca. \\
\hline & & & Características del sistema de drenaje. \\
\hline & \multirow{3}{*}{\multicolumn{2}{|c|}{ Actividad humana }} & Cortes o excavaciones. \\
\hline & & & Sobrecargas. \\
\hline & & & Deforestación. \\
\hline \multirow{2}{*}{$\begin{array}{l}\text { Dependientes del análisis } \\
\text { cuantitativo de la estabilidad }\end{array}$} & \multicolumn{2}{|c|}{ Superficie de rotura } & Profundidad. \\
\hline & \multicolumn{2}{|c|}{ Factor de seguridad } & Valor. \\
\hline
\end{tabular}

Fuente: Cuanalo, Oliva y González (2007).

Tabla 4. Intervalos y nivel de influencia de los factores de valuación

\begin{tabular}{lll}
\hline \multicolumn{1}{c}{ Factor de valuación } & \multicolumn{1}{c}{ Nivel de influencia } & \multicolumn{1}{c}{ Consideraciones } \\
\hline$<0,5$ & Bajo & No tiene problemas \\
\hline $0,5 \div 0,75$ & Medio & Requiere atención \\
\hline$>0,75$ & Alto & Atención urgente \\
\hline
\end{tabular}

Fuente: Cuanalo, Oliva y González (2007). 
Tabla 5. Evaluación cualitativa de la ladera objeto de estudio

\begin{tabular}{lc}
\hline \multicolumn{1}{c}{ Factor de valuación } & Valor \\
\hline Morfología y topografía & 0,45 \\
\hline Mecánica de suelos & 0,90 \\
\hline Hidrogeología & 0,75 \\
\hline Vegetación & 0,86 \\
\hline Actividad sísmica & 1,00 \\
\hline Erosión y socavación & 0,72 \\
\hline Actividad humana & 0,70 \\
\hline Análisis cuantitativo de estabilidad & 0,75 \\
\hline Factor general & 0,766 \\
\hline
\end{tabular}

Fuente: elaboración propia.

El factor de valuación general superior a 0,75 indica que los factores condicionantes y desencadenantes analizados tienen alto nivel de influencia sobre la inestabilidad de la ladera, por lo que la situación requiere de atención urgente (tabla 4).

\section{Instrumentación, monitoreo y control de movimientos}

Para conocer la evolución y características de los movimientos horizontales en la ladera, se acondicionaron los dos sondeos mecánicos del estudio geotécnico con tubería inclinométrica de ABS, la cual se solidariza al terreno mediante inyección de lechada de cemento.

Las lecturas inclinométricas realizadas durante un año permitieron definir los límites de la masa de terreno con mayor probabilidad de inestabilidad y la tendencia de los movimientos (figura 9) (Valbuena, García y Granados, 2017).

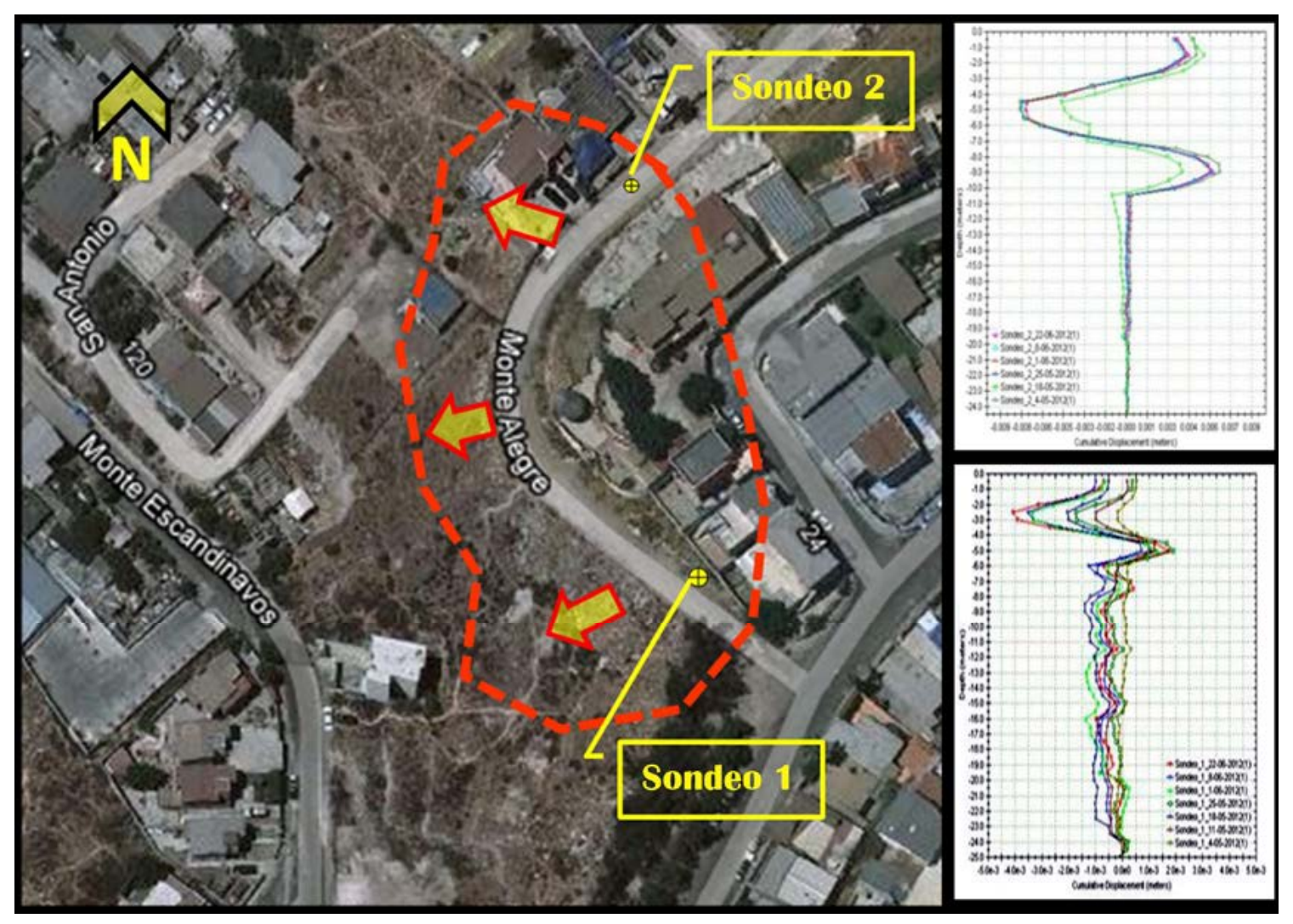

Figura 9. Área de terreno inestable y tendencias de movimiento

Fuente: elaboración propia. 


\section{Evaluación del riesgo}

\section{Amenaza}

La amenaza en la zona de estudio se evaluó considerando la probabilidad de que ocurran deslizamientos según los criterios: rango de frecuencia o periodo de retorno de los deslizamientos en la zona de estudio (tabla 6); y factores de seguridad obtenidos (tabla 7).

\section{- Rango de frecuencia}

La tabla 6 muestra una clasificación de la probabilidad de ocurrencia de deslizamientos, en función del rango de frecuencia o periodo de retorno de los mismos en la zona de estudio.

Tabla 6. Escala de probabilidades recomendadas para magnitudes o intensidades de deslizamientos

\begin{tabular}{lc}
\hline \multicolumn{1}{c}{ Término } & Rango de frecuencia (1/año) \\
\hline Muy alta probabilidad & $>1 / 20$ \\
\hline Alta & $1 / 100-1 / 20$ \\
\hline Media & $1 / 500-1 / 100$ \\
\hline Baja & $1 / 2500-1 / 500$ \\
\hline Muy Baja & $<1 / 2500$ \\
\hline
\end{tabular}

Fuente: Modificado de Hungr (1997).

Los registros de deslizamientos de laderas ocurridos en sitios cercanos al área de estudio, con similares características geológicas, geotécnicas, geomorfológicas y de actividad humana, indican que la frecuencia o periodo de retorno de deslizamientos en la zona es de uno en tres años (1/3), que es una probabilidad muy alta de ocurrencia.

El comportamiento de la probabilidad de ocurrencia será expresado matemáticamente mediante la ecuación (2).

$$
P_{x}=1-\left(1-P_{a}\right)^{x}
$$

Donde,

$P_{a}$ es la probabilidad para un período específico de tiempo (en nuestro caso, 1 año). En nuestra zona de estudio será 0,33.
$P_{x}$ es la probabilidad a largo plazo.

$x$ es un número dado de años.

- Factores de seguridad

Los factores de seguridad dinámicos obtenidos en el análisis cuantitativo de la estabilidad (tabla 2) indican que la amenaza de deslizamiento por efectos de la sismicidad en la mayoría de los perfiles estudiados es media-alta, según las escalas recomendadas en la tabla 7.

Tabla 7. Recomendaciones para evaluar la amenaza por deslizamientos

\begin{tabular}{lcc}
\hline Amenaza & $\begin{array}{c}\text { Factor de } \\
\text { seguridad estático }\end{array}$ & $\begin{array}{c}\text { Factor de seguridad } \\
\text { dinámico }\end{array}$ \\
\hline Baja & Superiores a 1,5 & Superiores a 1,15 \\
\hline Media & Entre 1,2 y 1,5 & Entre 1,0 y 1,15 \\
\hline Alta & Inferiores a 1,2 & Inferiores a 1,0 \\
\hline
\end{tabular}

Fuente: Modificado de Suarez (2012).

Los análisis anteriores permiten evaluar la probabilidad de ocurrencia de un deslizamiento en la zona como alta y considerar su valor igual a la probabilidad anual $\left(P_{a}=0,33\right)$.

\section{Vulnerabilidad}

Se refiere al grado de pérdida de los elementos en riesgo, como resultado de la ocurrencia probable de un deslizamiento en el área de estudio. Su valor representa el grado de pérdidas o nivel de daños potenciales de dichos elementos expuestos, y se expresa en una escala de 0 a 1.

Para evaluar la vulnerabilidad se consideraron las tres direcciones de posibles movimientos de la ladera y los daños que estos pueden producir en las edificaciones existentes en la zona de estudio, que constituyen los elementos expuestos (figura 10).

La tabla 10 muestra los elementos expuestos y sus vulnerabilidades físicas, obtenidas teniendo en cuenta el rango de pérdidas esperado por efecto espacial (Imiriland, 2002) y considerando la profundidad de las cimentaciones respecto a la profundidad del deslizamiento (Ragozin y Tikhvinsky, 2000). 


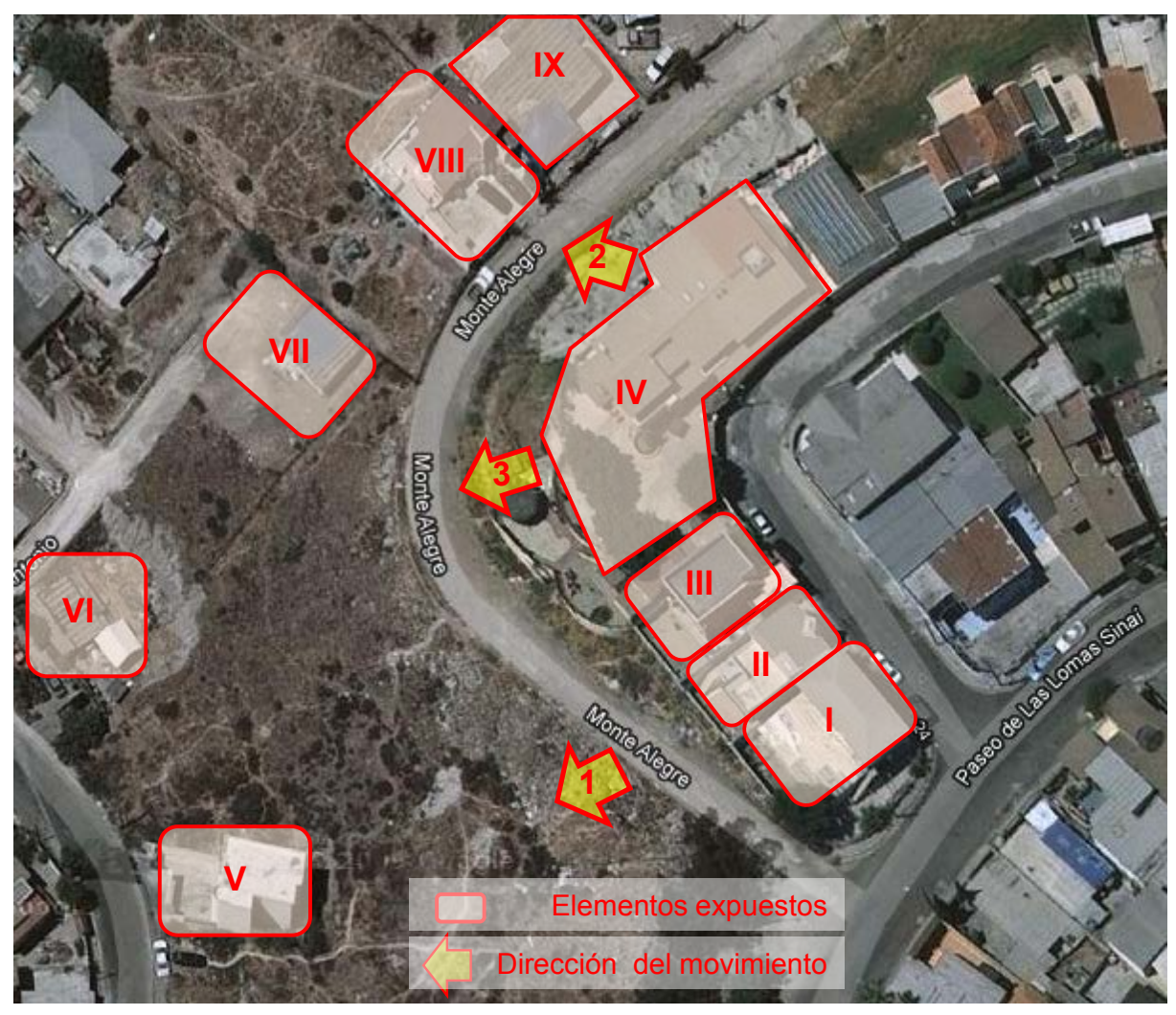

Figura 10. Elementos susceptibles a sufrir daños por los movimientos de la ladera

Fuente: elaboración propia.

Tabla 10. Vulnerabilidad de los elementos expuestos

\begin{tabular}{|c|c|c|}
\hline $\begin{array}{c}\text { Dirección del movimiento } \\
\text { (figura 10) }\end{array}$ & Elemento expuesto & Vulnerabilidad (V) \\
\hline \multirow[t]{4}{*}{ 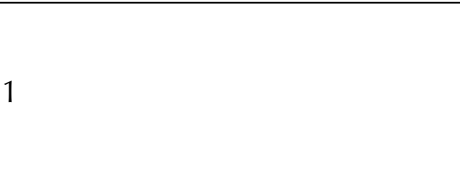 } & $\mathrm{I}$ & 1 \\
\hline & II & 1 \\
\hline & III & 1 \\
\hline & IV & 0,3 \\
\hline \multirow{4}{*}{2} & IV & 0,7 \\
\hline & VII & 0,5 \\
\hline & VIII & 1 \\
\hline & IX & 0,7 \\
\hline \multirow{6}{*}{3} & IV & 1 \\
\hline & III & 1 \\
\hline & VII & 0,5 \\
\hline & II & 0,5 \\
\hline & $\mathrm{VI}$ & 0,3 \\
\hline & $\mathrm{V}$ & 0,3 \\
\hline
\end{tabular}

Fuente: elaboración propia. 


\section{Riesgos}

Para evaluar el riesgo en la zona objeto de estudio, se consideraron: el riesgo específico $R(S)$; el valor del riesgo específico $R(S V)$; y el riesgo múltiple $R(M)$.

El riesgo específico (specific risk) es el grado de pérdidas o daños esperados en un elemento expuesto específico, como resultado del deslizamiento y se expresa según la ecuación (3).

$$
R(S)=P(H) * V
$$

Donde $V$ es la vulnerabilidad del elemento expuesto.

El valor del riesgo específico (value of specific risk) consiste en las pérdidas o daños en un elemento expuesto específico (excluyendo la vida humana) como resultado de la ocurrencia de un deslizamiento y se expresa según la ecuación (4).

$$
R(S V)=P(H) *(V \cdot E)
$$

Siendo $E$ el valor del elemento expuesto.

Cuantitativamente, $R(S V)$ se expresa en las mismas unidades que el término $V \times E$.

El riesgo múltiple (multiple risk) es el riesgo de más de un elemento expuesto ante un deslizamiento, o el de un elemento expuesto específico ante más de un movimiento.

En la tabla 11 se presenta la evaluación cuantitativa de los diferentes riesgos generados por el deslizamiento potencial de la ladera, en función de la amenaza y vulnerabilidad existentes.

En la figura 11 se presenta la estimación del comportamiento del riesgo en los elementos expuestos para la amenaza y vulnerabilidades existentes en el momento en que se hizo el estudio.

\begin{tabular}{|c|c|c|c|c|c|c|c|c|}
\hline $\begin{array}{l}\text { Dirección del } \\
\text { deslizamiento } \\
\text { (figura 10) }\end{array}$ & $\begin{array}{l}\text { Elemento } \\
\text { expuesto }\end{array}$ & $\begin{array}{c}\text { Amenaza } \\
P(H)\end{array}$ & $\begin{array}{l}\text { Vulnerabilidad } \\
\text { (V) }\end{array}$ & $\begin{array}{l}\text { Valor del elemento } \\
\text { expuesto } E \text { (USD) }\end{array}$ & $(V \times E)$ & $\begin{array}{c}\text { Riesgo } \\
\text { específico } \\
R(S)\end{array}$ & $\begin{array}{l}\text { Valor del riesgo } \\
\text { específico } R(S V)\end{array}$ & $\begin{array}{l}\text { Valor del riesgo } \\
\text { múltiple } R(M V)\end{array}$ \\
\hline & I & 0,3 & 1 & $\$ 122.726,54$ & $\$ 122.726,54$ & 0,3 & $\$ 36.817,96$ & \\
\hline \multirow[t]{5}{*}{1} & II & 0,3 & 1 & $\$ 122.726,54$ & $\$ 122.726,54$ & 0,3 & $\$ 36.817,96$ & \\
\hline & III & 0,3 & 1 & $\$ 87.137,00$ & $\$ 87.137,00$ & 0,3 & $\$ 26.141,10$ & $\$ 139.818,08$ \\
\hline & IV & 0,3 & 0,3 & $\$ 444.900,62$ & $\$ 133.470,18$ & 0,09 & $\$ 40.041,06$ & \\
\hline & IV & 0,3 & 0,7 & $\$ 444.900,62$ & $\$ 311.430,43$ & 0,21 & $\$ 93.429,13$ & \\
\hline & VII & 0,3 & 0,5 & $\$ 87.137,00$ & $\$ 43.568,50$ & 0,15 & $\$ 13.070,55$ & \\
\hline \multirow[t]{5}{*}{2} & VIII & 0,3 & 1 & $\$ 122.726,54$ & $\$ 122.726,54$ & 0,3 & $\$ 36.817,96$ & $\$ 161.616,41$ \\
\hline & IX & 0,3 & 0,7 & $\$ 87.137,00$ & $\$ 60.995,90$ & 0,21 & $\$ 18.298,77$ & \\
\hline & IV & 0,15 & 1 & $\$ 444.900,62$ & $\$ 444.900,62$ & 0,15 & $\$ 66.735,09$ & \\
\hline & III & 0,15 & 1 & $\$ 87.137,00$ & $\$ 87.137,00$ & 0,15 & $\$ 13.070,55$ & \\
\hline & VII & 0,15 & 0,5 & $\$ 87.137,00$ & $\$ 43.568,50$ & 0,075 & $\$ 6.535,28$ & $\$ 103.387,74$ \\
\hline \multirow[t]{3}{*}{3} & II & 0,15 & 0,5 & $\$ 122.726,54$ & $\$ 61.363,27$ & 0,075 & $\$ 9.204,49$ & \\
\hline & $\mathrm{VI}$ & 0,15 & 0,3 & $\$ 87.137,00$ & $\$ 26.141,10$ & 0,045 & $\$ 3.921,17$ & \\
\hline & $\mathrm{V}$ & 0,15 & 0,3 & $\$ 87.137,00$ & $\$ 26.141,10$ & 0,045 & $\$ 3.921,17$ & \\
\hline
\end{tabular}

Tabla 11. Evaluación de riesgos en la zona de estudio

Fuente: elaboración propia. 

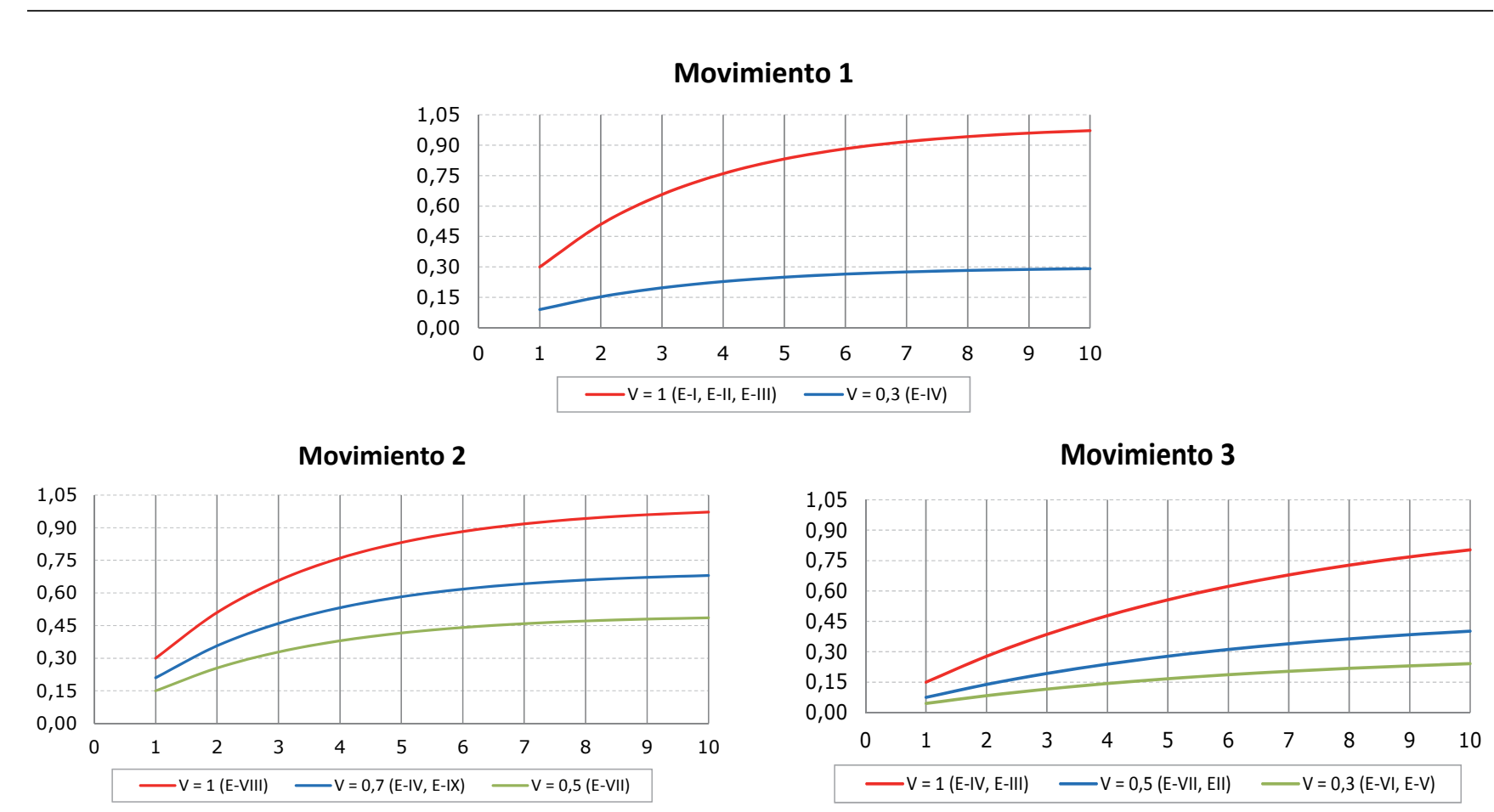

Figura 11. Comportamiento del riesgo de los elementos expuestos en las tres direcciones de movimiento esperadas

Fuente: elaboración propia.

\section{CONCLUSIONES}

La frecuencia con que se presentan deslizamientos en sitios cercanos a la zona de estudio y con características geológicas, geotécnicas, geomorfológicas y de actividad humana similares, indican una alta probabilidad de ocurrencia de estos fenómenos. Lo anterior se confirma y valida en el análisis cuantitativo de la estabilidad, donde se obtienen factores de seguridad por debajo de los valores considerados aceptables; y con los resultados del análisis cualitativo, donde los factores de valuación indican que la ladera es inestable y requiere de atención urgente.

Los resultados del análisis de estabilidad en diferentes perfiles y las lecturas inclinométricas en los sondeos, indican que la ladera tiene tres direcciones posibles de movimiento, las cuales influyen de manera importante en la evaluación del riesgo.
Las edificaciones ubicadas en la zona con mayores probabilidades de sufrir daños, constituyen elementos expuestos al deslizamiento y su vulnerabilidad física varía entre 0,3 y 1 , dependiendo del tipo y distribución espacial de las edificaciones, su estado de integridad estructural, profundidad de las cimentaciones en relación a la profundidad del deslizamiento, y del volumen probable de la masa de terreno inestable.

La existencia de una alta probabilidad de deslizamiento, tres posibles direcciones de movimiento y varias edificaciones expuestas al impacto de los mismos, genera riesgos múltiples altos que elevan significativamente el nivel de riesgo total en la zona.

Los resultados del estudio no solo permitieron identificar los factores condicionantes y desencadenantes de la inestabilidad en la ladera $y$ sus influencias, sino que constituyen una valiosa 
herramienta de trabajo para las instituciones y autoridades que tienen como misión reducir el riesgo por deslizamientos, como forma de prevenir, mitigar y corregir eventos generadores de desastres en las comunidades expuestas.

\section{FINANCIAMIENTO}

El artículo se deriva del proyecto de investigación "Diagnóstico, evaluación y modelación del riesgo por inestabilidad de laderas en el sector norte del Fraccionamiento Lomas Conjunto Residencial de la Delegación La Mesa, en la ciudad de Tijuana, Baja California, México".

Entidad financiadora: Ayuntamiento de Tijuana.

Entidad que avala: Dirección Municipal de Protección Civil de Tijuana.

\section{REFERENCIAS}

Acosta Chang, J., Arellano Zepeda, G., Ruiz Cruz, E., Mendoza Garcilazo, L., Reyes Serrano, R., \& Rocha Guerrero, E. (2009). Microzonación sísmica en la zona urbana de Tijuana, BC. México: CICESE, Dirección Estatal de Protección Civil, FOPREDEN.

Correa Leguizamón, M., y Alfaro Castillo, A. (2011). Necesidad de la revisión de los estudios de amenaza sísmica a raíz del sismo de Tohoku de 2011. Tecnura, 15(30), 82-93. https://doi.org/10.14483/ udistrital.jour.tecnura.2011.2.a08

Cuanalo C., O.A., Oliva G., A.O. y González N., C. (2007). Estabilidad de laderas. Análisis mediante factores de valuación. Revista IngeoPres, (164), 38-44.

Cuanalo C., O.A., Oliva G., A.O. y Gallardo A., R.J. (2012). Inestabilidad de laderas: Procesos constructivos de estabilización. Análisis mediante factores de valuación. España: Editorial Académica Española.

Cruz-Castillo, M. (2002). Catálogo de las fallas regionales activas en el norte de Baja California, México. GEOS, Unión Geofísica Mexicana, 22, 37-42.

Cyment, D. (1979). The Human settlements on the San Andreas Fault. Recuperado de https://
books.google.com.co/books/about/The_Human_settlements_on_the_San_Andreas. html?id=UBVOAQAAIAAJ\&redir_esc=y

Fondo Nacional para la Prevención de Desastres Naturales. (2009). Microzonificación sísmica de Tijuana. Tijuana. Recuperado de http://www.proteccioncivil.tijuana.gob.mx/pdf/planes/estudios/Informe\%20 Final\%20de\%20Microzonificacion \%20Sismica\%20de\%20Tijuana\%202009.pdf

Hungr, O. (1997). Some methods of landslide hazard intensity mapping. En Landslide risk assessment. Proceedings of the international workshop on landslide risk assessment (pp. 215-226). Honolulu: Balkema, Rotterdam.

Imiriland. (2002). Identification and Mitigation of Large Landslide Risks in Europe. Project Imiriland D 16. Relevant criteria to assess vulnerability and risk. Recuperado http://drm.cenn.org/training_materials/ Session\%2005\%20Vulnerability\%20assessment. pdf

IUGS Working Group on Landslides, \& Committee on Risk Assessment. (1997). Quantitative risk assessment for slopes and landslides-The state of the art. En Landslide risk assessment. Proceedings of the international workshop on landslide risk assessment (pp. 15-23). Honolulu: Balkema, Rotterdam

Morgenetern, N. R. (1997). Toward landslide risk assessment in practice. En Landslide risk assessment. Proceedings of the international workshop on landslide risk assessment (pp. 15-23). Honolulu: Balkema, Rotterdam.

Oliva G., A.O. et al. (2014). Hillside instability in the Tijuana metropolitan area. Analysis of landslideprovoked building collapse. Engineering Failure Analysis, 46, 166-178. https://doi.org/10.1016/j. engfailanal.2014.08.004

Ragozin, A.L. y Tikhvinsky I.O. (2000). Landslide Hazard, vulnerability and risk assessment. En E. Bromhead (ed.), Landslides in Research, Theory and Practice (pp. 1257-1262). Cardiff, Gales: Thomas Telford.

Suarez, J. (2012). Deslizamientos. Análisis Geotécnico. Recuperado de http://www.erosion.com.co/deslizamientos-tomo-i-analisis-geotecnico.html 
Suarez-Vidal, F., Armijo, R., Morgan, G., Bodin, P., \& Gastil, R. G. (1991). Framework of Recent and Active Faulting in Northern Baja California: Chapter 16: Part III. Regional Geophysical and Geology (pp. 285-300). Tulsa, Oklahoma: AAPG Memoir.
García Ubaque, C., Valbuena Porras, S., \& Granados Soler, M. (2017). Metodología para el monitoreo estructural y patológico de viviendas afectadas por deslizamientos. Tecnura, 21(52), 79-87. https://doi. org/10.14483/udistrital.jour.tecnura.2017.2.a06

\section{(9) $(1) \Theta \Theta$}

